\title{
Experimental observation of к-phase formation sequences by in-situ synchrotron diffraction
}

\author{
C. Drouven ${ }^{\mathrm{a}, *}$, B. Hallstedt ${ }^{\mathrm{b}}$, W. Song ${ }^{\mathrm{a}}$, W. Bleck ${ }^{\mathrm{a}}$
}

${ }^{a}$ Steel Institute, Intzestraße 1, 52072 Aachen, Germany

${ }^{\mathrm{b}}$ Institute for Materials Applications in Mechanical Engineering, Augustinerbach 4, 52062 Aachen

*Corresponding author. E-Mail: carsten.drouven@iehk.rwth-aachen.de

\section{Abstract}

In-situ annealing experiments using synchrotron diffraction depict the separation of $\{100\}$-superlattice peaks ascribed to C-enrichment of pre-existing, $\mathrm{C}$-lean $\mathrm{L}^{\prime} 1_{2}$-ordered structures into $\mathrm{C}$-rich $\kappa$-carbides of alike crystal structure during heating of a high-Al lightweight steel. The peak separation is the first experimental in-situ investigation of the transformation sequence during $\mathrm{Fe}_{3} \mathrm{AlC}$-phase formation. A detailed analysis of the lattice parameter evolution and thermal expansion behavior was carried out to validate the assignment of phases by comparison with theoretical calculations and available experimental data.

Keywords: synchrotron diffraction, intermetallics, ordering, phase transformation

\section{Introduction}

The addition of noteworthy amounts of aluminum to steel yields a twofold advantage by reducing the overall density of steel whilst increasing its strength due to the formation of intermetallic phases. Latter strengthening contribution requires a profound knowledge of the phase transformation involving the precipitation of intermetallic phases. The $\mathrm{Fe}_{3} \mathrm{AlC}$ - or $\kappa$-phase can be stabilized in high-Al alloys either within a face-centered cubic (fcc) austenite or body-centered cubic (bcc) ferrite matrix. The beneficial contribution of the $\kappa$-phase to the mechanical properties of fully austenitic high-manganese steels has led to research on the underlying phase transformations [1,2].

Spinodal decomposition was proposed to occur during phase transformation from austenite $(\gamma)$ to the $\kappa$ phase [3,4]. The decisive distinction between disordered $\gamma$ and the ordered configurations lies in a transition from fcc to simple cubic structures and thus an additional superlattice reflection. The $\kappa$-phase exhibits a $\mathrm{L}^{\prime} 1_{2}$-crystal structure, wherein $\mathrm{Fe}$ and $\mathrm{Al}$ are ordered on simple cubic sublattices and $\mathrm{C}$ occupies the center of the lattice structure, forming a perovskite-like structure. $\{100\}$-superreflection corresponding to a single crystal lattice structure were identified in Fe-Mn-Al-C steels upon water quenching $[5,6]$. A possible $\kappa$-formation sequence is shown schematically in Figure 1. Possible reaction sequences for first-order order-disorder transformations are discussed in detail by Soffa and Laughlin [7]. Therein, a disordered fcc state is cooled from high temperatures and undergoes in full long-range ordering by diffusion over the range of a few atomic distances. Following, this ordered structure separates into a disordered fcc and a L' $1_{2}$-structured $\kappa$-phase, possibly by spinodal decomposition. The aforementioned reaction sequence was also suggested by Tan et al. [8] for Ni-base superalloys. 
The first formed ordered phase and the equilibrium $\kappa$-phase both exhibit a $\mathrm{L}^{\prime} 1_{2}$ crystal structure, whereby the difference lies in the Al- and C-concentration (c.f. Figure 1). Hence, the two types of configurations are denoted as C-lean and C-rich $\kappa$-phase, respectively.

The lack of in-situ studies on the $\kappa$-phase formation constitute uncertainties regarding the $\kappa$-phase transformation sequence and its underlying kinetics during $\kappa$-phase formation. In the current work, the first in-situ experimental observation of the $\kappa$-formation was conducted by synchrotron diffraction during heating of a high-Al alloyed lightweight steel.

\section{Experimental section}

The two-phase alloy X100AlMnNi 10-7-6 was vacuum induction melted into ingots. The alloy composition was determined by wet-chemical analysis and X-ray fluorescence. The chemical composition is provided in Table 1 . Homogenization was conducted at $1200{ }^{\circ} \mathrm{C}$ for $5 \mathrm{~h}$ prior to opendie hot forging at $1200^{\circ} \mathrm{C}$ with a total strain of 0.7 and subsequent water quenching. The slabs of dimension $40 \mathrm{~mm} \times 40 \mathrm{~mm} \times 320 \mathrm{~mm}$ were solution annealing at $1200{ }^{\circ} \mathrm{C}$ for $2 \mathrm{~h}$ followed by water quenching.

Synchrotron X-ray diffraction was carried out in the high-resolution powder diffraction beamline P02.1 at DESY, Hamburg. A fixed wavelength of approx. $0.207 \AA$ was used to acquire diffraction pattern on a PerkinElmer XRD1621 fast area detector. The $2 \Theta$-range expands from $\sim 2^{\circ}-16^{\circ}$. In-situ heating was conducted in a ceramic heating oven using sealed quartz capillaries. Heating rates of $15^{\circ} \mathrm{C} / \mathrm{min}$ were employed until an isothermal holding temperature of $800{ }^{\circ} \mathrm{C}$ was reached. During heating, diffraction patterns are acquired in steps of $50^{\circ} \mathrm{C}$. Image acquisition during holding is divided into steps of $30 \mathrm{~s}$. Data analysis is carried out using Fit2D [9] for conversion of 2D patterns into 1D intensity profiles. The subsequent Rietveld analysis is performed using the open source software 'Materials Analysis Using Diffraction' [10]. The $2 \Theta$-range was reduced to $\sim 10^{\circ}$, limiting data analysis to completely acquired Debye Scherrer rings.

\section{Results and discussion}

The diffraction profile of the duplex alloy after solution annealing is displayed in Figure 2a. $\{111\}_{f c c^{-}}$ and $\{110\}_{b c c}$-peaks confirm the presence of austenite and ferrite. In addition, ordered phases of the $\mathrm{L}^{\prime} 1_{2^{-}}$and B2-type are present as visualized in Figure $2 \mathrm{~b}$ by an enlarged view of the superlattice reflections corresponding to the $\{100\}_{L^{\prime} 1_{2}}$ - and $\{110\}_{L^{\prime} 1_{2}}$-peak as well as the $\{100\}_{B 2}$-peak.

Figure $2 \mathrm{c}$ displays the evolution of the $\{100\}_{L^{\prime} 1_{2}}$-superlattice reflection during heating from $200{ }^{\circ} \mathrm{C}$ to $800{ }^{\circ} \mathrm{C}$. The peak intensity increases during heating up to $600{ }^{\circ} \mathrm{C}$, indicating an increase in volume fraction of $\mathrm{L}^{\prime} 1_{2}$-ordered phases. Simultaneously, the peak position shifts continuously towards lower angles due to thermal expansion of the lattice structure. Heating above $600{ }^{\circ} \mathrm{C}$ results in a pronounced peak separation of the initial peak by a secondary reflection at lower diffraction angles (c.f. Figure 2c). 
The considerable shift in peak position of the secondary reflection accentuates the occurrence of a phase transformation. The phase transformation is accompanied by a decrease in volume fraction of the initial phase. At $700{ }^{\circ} \mathrm{C}$, the majority share of the superlattice reflection has transformed into the newly formed configuration. The transformation is concluded upon reaching $750{ }^{\circ} \mathrm{C}$ and thus occurs in approximately 5 minutes.

Figure 3a depicts the time-resolved evolution of the lattice parameter corresponding to the diverse superlattice reflections. During heating, the room temperature lattice parameter yields $3.672 \AA$ and increases up to $3.735 \AA$. The ordering transformation commences at $650{ }^{\circ} \mathrm{C}$ and results in an increase of lattice parameter to an overall average of $3.821 \AA \pm 0.039 \AA$ (errors as standard error).

The schematic reaction sequence in Figure 1 suggests that fcc- and $\kappa$-phase may reach their respective equilibrium state thermodynamically. In contrast, the matrix-particle coherency between the fcc- and $\kappa$ phase restrains the phases to maintain an alike lattice parameter. However, the equilibrium lattice parameters are different, with $\kappa$-phase having a clearly larger lattice parameter. The lattice parameter of the $\kappa$-phase increases with, in particular, the $\mathrm{C}$ content. Therefore, the coherently constrained $\kappa$-phase has a lower $\mathrm{C}$ content than the equilibrium $\kappa$-phase. Consequently, the observed peak separation of the superlattice reflection is ascribed to the transformation from the C-lean to the C-rich $\kappa$-phase. Both configurations evoke superlattice reflections, while an enrichment of the C-lean $\kappa$-phase is anticipated to induce a prompt increase in lattice parameter. The assignment of the peak separation to C-enrichment of the C-lean $\kappa$-phase is corroborated by the fast kinetics associated with the high $\mathrm{C}$ mobility at elevated temperatures. Under latter consideration, the completion of ordering and the subsequent phase separation to a C-rich $\kappa$-phase is considered plausible under certain cooling conditions, thus suppressing the measurement of the C-lean $\kappa$-phase.

The $\kappa$-phase frequently exhibits an under-stoichiometric concentration of $\mathrm{Al}$ and $\mathrm{C}$ [11]. In general, the enrichment of fcc and its related ordered structure with $\mathrm{C}$ is expected to cause an increase in lattice constant $[12,13]$. The $\mathrm{C}$-enrichment of the $\kappa$-phase with respect to its parent fcc-phase is well documented by atom probe tomography measurements $[12,14]$. The C-enrichment induces an increase in lattice parameter by approximately $0.09 \AA$ at $650^{\circ} \mathrm{C}$. The thermal expansion of the $\mathrm{C}$-lean $\mathrm{L}^{\prime} 1_{2}$-phase was investigated in detail in Figure 3b. Its room temperature lattice parameter of about $3.672 \AA$ increases approximately linear with temperature and the thermal expansion is approximated empirically to:

$$
a_{L^{\prime} 12}^{C-\text { lean }}(T)=(3.66505 \pm 0.00285) \AA+\left(8.06418 * 10^{-5} \pm 0.66981 * 10^{-5}\right) \AA / K^{*} T \quad \text { Equation } 1
$$

Experimental measurements $[12,13,15,16]$ and theoretical calculations $[11,17]$ of the room temperature lattice constant associated with the C-rich $\kappa$-phase yield a range from $3.72 \AA-3.78 \AA$. Under assumption of an identical thermal expansion, the room temperature lattice parameter of the C-rich $\kappa$-phase $a_{L^{\prime} 12}^{C-r i c h}(R T)$ would yield approximately $3.756 \AA$ by recalculating $a_{L^{\prime} 12}^{C-r i c h}\left(800^{\circ} \mathrm{C}\right)$ assuming the thermal expansion coefficient of the C-lean $\kappa$-phase in Equation 1. 
The lack of experimental observations of the $\mathrm{C}$-lean $\kappa$-phase impedes a validation of the measured lattice parameter. Nevertheless, the C-lean $\kappa$-phase resembles in first approximation the $\mathrm{C}$-free $\mathrm{Fe}_{3} \mathrm{Al}-\mathrm{L} 1_{2}$ phase. Maugis [18] used ab-initio calculations to determine the room temperature lattice parameter of the $\mathrm{Fe}_{3} \mathrm{Al}-\mathrm{L} 1_{2}$ and $\mathrm{Fe}_{3} \mathrm{AlC}-\mathrm{L}^{\prime} 1_{2}$ as $3.65 \AA$ and $3.75 \AA$, respectively. Similar calculations of theoretical lattice parameter of $\mathrm{L} 1_{2}-\mathrm{Fe}_{3} \mathrm{Al}$ and $\mathrm{L}^{\prime} 1_{2}-\mathrm{Fe}_{3} \mathrm{AlC}$ yielded differences of $0.04 \AA-0.10 \AA$ [17,19]. The accordance of theoretical calculations and experimental observations in lattice parameter with respect to absolute values and differences confirms the transformation sequence from disordered fcc phase to an ordered, C-lean $\kappa$-phase followed by a phase separation into disordered fcc and C-rich $\kappa$-phase.

\section{Conclusions}

In-situ synchrotron diffraction facilitates the identification of the $\kappa$-phase formation sequence, wherein the peak separation of superlattice reflections corresponding to a $\mathrm{L}^{\prime} 1_{2}$-crystal structure was linked to the development of a C-rich $\kappa$-phase from an ordered C-lean $\kappa$-precursor. The allocation of phases was validated by comparison of lattice parameter to theoretical calculations and experimental data. The observed transformation sequence suggests a prior ordering of the disordered fcc-phase into a longrange ordered, C-lean $\kappa$-precursor, which subsequently undergoes phase separation into a disordered fcc- and C-rich $\kappa$-phase.

\section{Acknowledgements}

The financial support of the Deutsche Forschungsgemeinschaft (DFG), Grant no. SO1431 and the support by the DESY synchrotron beamline P02.1 is gratefully acknowledged.

\section{References}

[1] K.-G. Chin, H.-J. Lee, J.-H. Kwak, J.-Y. Kang, B.-J. Lee, J. Alloys Compd. 505 (2010) 217-223.

[2] W. Song, W. Zhang, J. von Appen, R. Dronskowski, W. Bleck, Steel Res. Int. 86 (2015) 11611169.

[3] W.K. Choo, J.H. Kim, J.C. Yoon, Acta Mater. 45 (1997) 4877-4885.

[4] W.-C. Cheng, C.-Y. Cheng, C.-W. Hsu, D.E. Laughlin, Mater. Sci. Eng. A 642 (2015) 128-135.

[5] M.C. Ha, J.-M. Koo, J.-K. Lee, S.W. Hwang, K.-T. Park, Mater. Sci. Eng. A 586 (2013) 276-283.

[6] C.S. Wang, C.N. Hwang, C.G. Chao, T.F. Liu, Scr. Mater. 57 (2007) 809-812.

[7] W.A. Soffa, D.E. Laughlin, Acta Metall. 37 (1989) 3019-3028.

[8] X.P. Tan, D. Mangelinck, C. Perrin-Pellegrino, L. Rougier, C.-A. Gandin, A. Jacot, D. Ponsen, V. Jaquet, Metall. Mater. Trans. A 45 (2014) 4725-4730.

[9] A.P. Hammersley, Eur. Synchrotron Radiat. Facil. Intern. Rep. ESRF97HA02T 68 (1997) 58.

[10]M. Ferrari, L. Lutterotti, J. Appl. Phys. 76 (1994) 7246-7255.

[11]M.J. Yao, P. Dey, J.-B. Seol, P. Choi, M. Herbig, R.K.W. Marceau, T. Hickel, J. Neugebauer, D. Raabe, Acta Mater. 106 (2016) 229-238.

[12]C.W. Kim, S.I. Kwon, B.H. Lee, J.O. Moon, S.J. Park, J.H. Lee, H.U. Hong, Mater. Sci. Eng. A 673 (2016) 108-113.

[13]L.N. Bartlett, D.C.V. Aken, J. Medvedeva, D. Isheim, N.I. Medvedeva, K. Song, Metall. Mater. Trans. A 45 (2014) 2421-2435.

[14] J.-B. Seol, D. Raabe, P. Choi, H.-S. Park, J.-H. Kwak, C.-G. Park, Scr. Mater. 68 (2013) 348-353.

[15]K. Sato, K. Tagawa, Y. Inoue, Mater. Sci. Eng. A 111 (1989) 45-50.

[16]K. Sato, K. Tagawa, Y. Inoue, Metall. Trans. A 21 (1990) 5-11.

[17]D. Connétable, P. Maugis, Intermetallics 16 (2008) 345-352.

[18]P. Maugis, J. Lacaze, R. Besson, J. Morillo, Metall. Mater. Trans. A 37 (2006) 3397-3401. 
[19]B.V. Reddy, S.C. Deevi, Mater. Sci. Eng. A 329-331 (2002) 395-401. 
Table 1: Chemical composition of the multi-phase alloy.

\begin{tabular}{|c|c|c|c|c|c|c|c|c|}
\hline Fe-10Al-7Mn-6Ni-1C & $\mathbf{C}$ & $\mathbf{M n}$ & $\mathbf{S i}$ & $\mathbf{P}$ & $\mathbf{S}$ & $\mathbf{N}$ & $\mathbf{A l}$ & $\mathbf{N i}$ \\
\hline wt.-\% & 0.95 & 6.8 & 0.025 & 0.012 & 0.011 & 0.012 & 10.1 & 6.1 \\
\hline
\end{tabular}

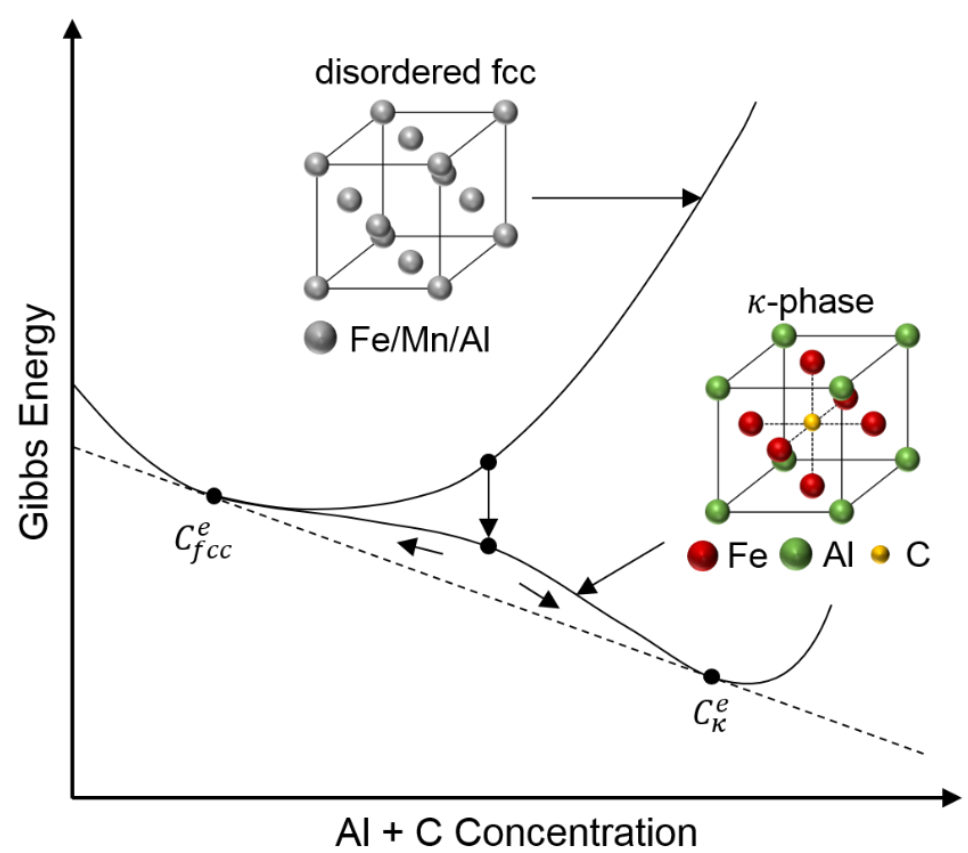

Figure 1 Schematical illustration of Gibbs energy curves in dependence of the Al- and C-concentration and the crystal structures of participating phases.

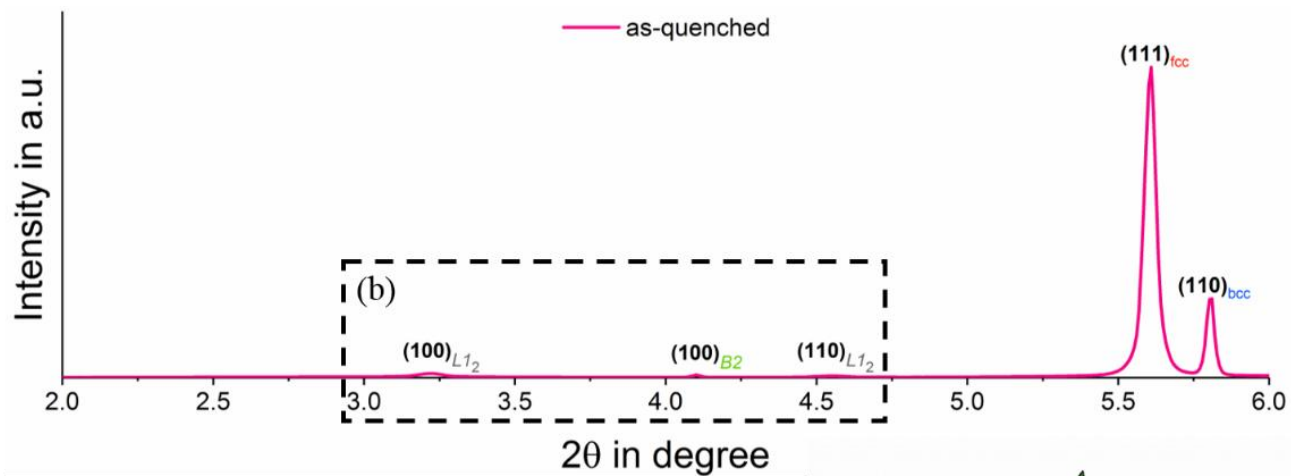

(a)

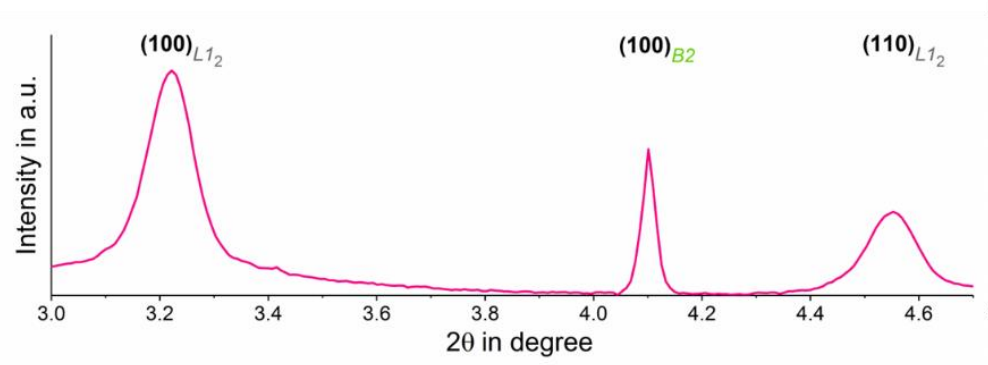

(b)

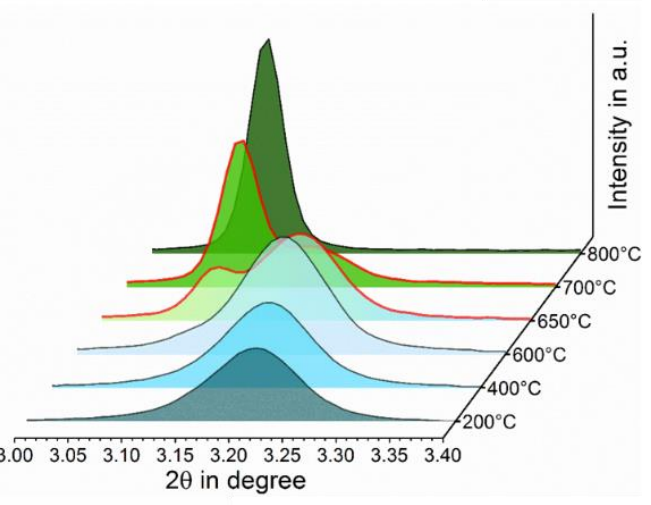

(c)

Figure 2: Synchrotron diffraction profile of the duplex alloy in the as-quenched condition (a) with an enlarged view (b) of superreflections corresponding to $\mathrm{L1}_{2}$ - and B2-structures. (c) Evolution of $\{100\}_{L^{\prime} 1_{2}}{ }^{-- \text {superlattice reflection during }}$ heating from $200{ }^{\circ} \mathrm{C}$ to isothermal holding temperature of $800{ }^{\circ} \mathrm{C}$. 


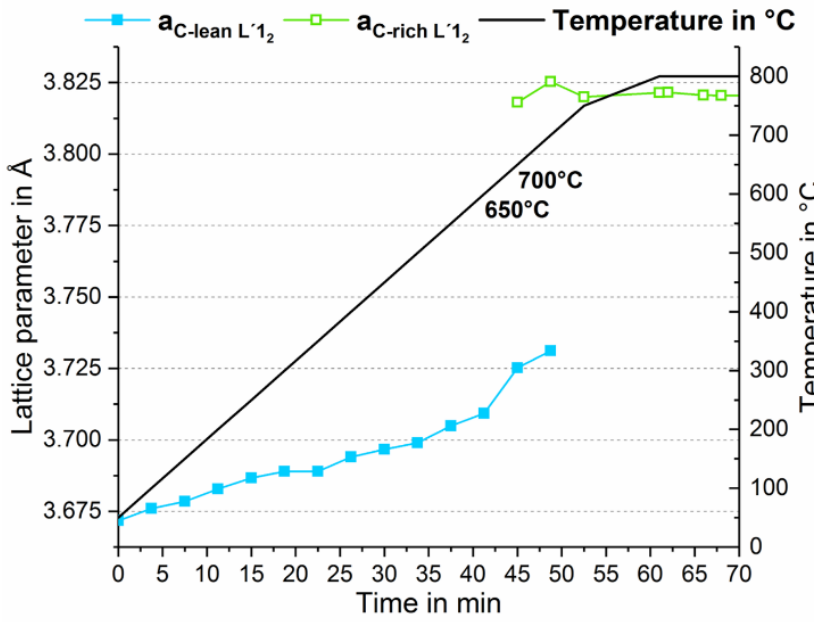

(a)

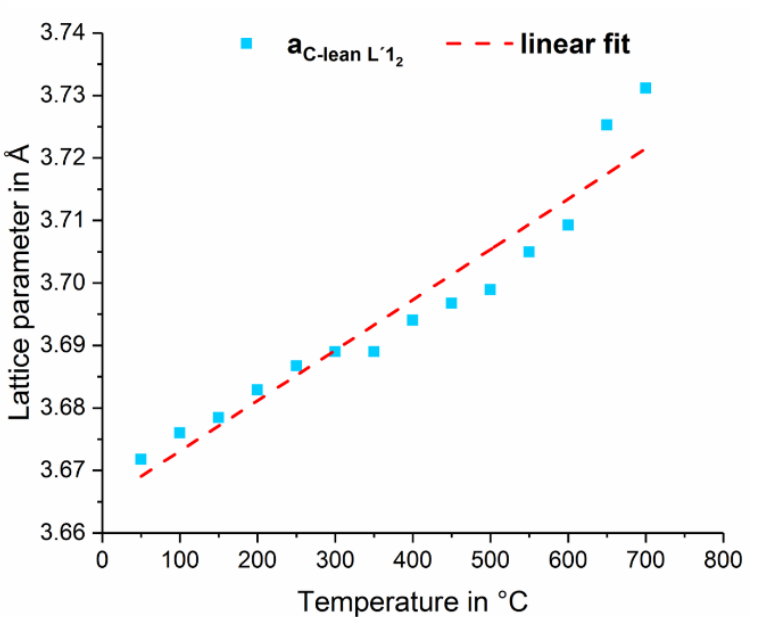

(b)

Figure 3 Evolution of the lattice parameter during heating of the C-lean and C-rich $L^{\prime} \mathbf{1}_{2}$-structure (a) and thermal expansion of the $C$-lean $L^{\prime} 1_{2}$-phase. 